\title{
Thermodynamics of Weakly Measured Quantum Systems
}

\author{
Jose Joaquin Alonso, ${ }^{1}$ Eric Lutz, ${ }^{1}$ and Alessandro Romito ${ }^{2}$ \\ ${ }^{1}$ Department of Physics, Friedrich-Alexander-Universität Erlangen-Nürnberg, D-91058 Erlangen, Germany \\ ${ }^{2}$ Dahlem Center for Complex Quantum Systems, FU Berlin, D-14195 Berlin, Germany
}

(Received 7 August 2015; published 25 February 2016)

\begin{abstract}
We consider continuously monitored quantum systems and introduce definitions of work and heat along individual quantum trajectories that are valid for coherent superposition of energy eigenstates. We use these quantities to extend the first and second laws of stochastic thermodynamics to the quantum domain. We illustrate our results with the case of a weakly measured driven two-level system and show how to distinguish between quantum work and heat contributions. We finally employ quantum feedback control to suppress detector backaction and determine the work statistics.
\end{abstract}

DOI: 10.1103/PhysRevLett.116.080403

Thermodynamics is, at its heart, a theory of work and heat. The first law is based on the realization that both quantities are two forms of energy and that their sum is conserved. At the same time, the fact that entropy, defined as the ratio of reversible heat and temperature, can only increase in an isolated system is an expression of the second law [1,2]. In classical thermodynamics, work is defined as the change of internal energy in an isolated system, $W=\Delta U$, while heat is introduced as the difference, $Q=\Delta U-W$, in a nonisolated system. Thermal isolation is thus crucial to distinguish $W$ from $Q$. In the last decades, stochastic thermodynamics has successfully extended the definitions of work and heat to the level of single trajectories of microscopic systems [3]. In this regime, thermal fluctuations are no longer negligible and the laws of thermodynamics have to be adapted to fully include them. The second law has, for instance, been generalized in the form of fluctuation theorems that quantify the occurrence of negative entropy production [4]. A particular example is the Jarzynski equality, $\langle\exp (-\beta W)\rangle=$ $\exp (-\beta \Delta F)$, which allows the determination of equilibrium free energy differences $\Delta F$ from the nonequilibrium work statistics in systems at initial inverse temperature $\beta$ [5]. The laws of stochastic thermodynamics have been verified in a large number of different experiments; see Refs. [6,7] and the review [8].

The current challenge is to extend the principles of thermodynamics to include quantum effects which are expected to dominate at smaller scales and colder temperatures. Some of the unsolved key issues concern the correct definition of quantum work and heat, means to distinguish between the two quantities owing to the blurring effect of quantum fluctuations, and the proper clarification of the role of quantum coherence. A variety of approaches have been suggested to tackle these problems [9-20], and quantum work statistics has been measured in isolated systems in two pioneering experiments using NMR [21] and trapped ions [22]. A new approach may emerge from the possibility of weakly monitoring quantum systems. Recently, individual quantum trajectories of a superconducting qubit in a microwave cavity have been observed using weak measurements $[23,24]$. These measurements only slightly disturb quantum systems owing to the weak coupling to the measuring device [25]. They hence allow us to gain information about states without projecting them into eigenstates. They have been successfully employed to explain quantum paradoxes [26], detect and amplify weak signals [27,28], determine a quantum virtual state [29], and directly measure a wave function [30]. Motivated by the two experiments $[23,24]$, we here investigate the first and second law for continuously monitored quantum systems and aim at developing a quantum stochastic thermodynamics based on quantum trajectories. Such an extension faces several technical difficulties. First, since a weakly measured system can be in a coherent superposition of energy eigenstates, energy is not a well-defined concept along a single quantum trajectory. Furthermore, even in the absence of an external environment, a continuously monitored quantum system is not isolated and the detector backaction, albeit small, will perturb its dynamics [25]. As a result, its time evolution will be nonunitary and energy, in the form of heat, will be exchanged with the detector.

In the following, we introduce suitable and consistent definitions of work and heat contributions to the quantum stochastic evolution of a weakly measured system that is externally driven. We use these definitions to determine the distributions of quantum work and heat for a two-level system, and to demonstrate the general validity of the Jarzynski equality, hence of the second law. We finally use the tools of quantum feedback control [31] to suppress detector backaction and thus effectively achieve thermal isolation of the system. This provides a practical scheme to experimentally test our definitions of work and heat along individual quantum trajectories.

Quantum work and heat.-We consider a system with time-dependent Hamiltonian $H_{t}$ that is initially in a thermal 
state at inverse temperature $\beta, \rho_{0}=\exp \left(-\beta H_{0}\right) / Z_{0}$, where $Z_{0}$ is the partition function. The system is driven by an external parameter $\lambda_{t}$ during a time $\tau$. At the ensemble level, quantum work and heat are introduced by considering an infinitesimal variation of the mean energy, $U=\langle H\rangle=$ $\operatorname{tr}\left\{\rho_{t} H_{t}\right\}[32,33]$ :

$$
d U=\operatorname{tr}\left\{\rho_{t} d H_{t}\right\}+\operatorname{tr}\left\{d \rho_{t} H_{t}\right\}=\delta W+\delta Q .
$$

Heat is further related to entropy $S=-k \operatorname{tr}\left\{\rho_{t} \ln \rho_{t}\right\}$ via $\delta Q=T d S[32,33]$. For an isolated system with unitary dynamics heat vanishes, since $d S=0$, and therefore $d U=$ $\delta W$ in agreement with classical thermodynamics $[1,2]$. Heat therefore appears to be fundamentally associated with the nonunitary part of the dynamics.

At the level of individual realizations, energy is a stochastic quantity owing to thermal and quantum fluctuations. The distribution $p(u)$ of the total energy change $u$ may be determined by performing projective measurements $\Pi_{n}$ and $\Pi_{m}$, with outcomes $E_{n}^{0}$ and $E_{m}^{\tau}$, at the beginning and at the end of the driving protocol $[9,34]$,

$$
p(u)=\sum_{m, n} P_{m, n}^{\tau} P_{n}^{0} \delta\left(u-\Delta E_{m, n}\right) .
$$

Here, $P_{n}^{0}=\operatorname{tr}\left\{\Pi_{n} \rho_{0}\right\}$ denotes the probability of the eigenvalue $E_{n}^{0}, P_{m, n}^{\tau}=\operatorname{tr}\left\{\Pi_{m} \rho_{n, \tau}\right\}$ the transition probability from state $n$ to $m$, with $\rho_{n, \tau}$ being the time evolved projected density operator $\rho_{n, 0}=\Pi_{n} \rho_{0} \Pi_{n} / P_{n}^{0}$, and $\Delta E_{m, n}=E_{m}^{\tau}-$ $E_{n}^{0}$ the energy difference. For unitary dynamics, $p(u)$ reduces to the work distribution $p(W)$, but, in general, Eq. (2) does not allow us to distinguish work from heat. In the following, we generalize Eq. (2) and identify work and heat for a weakly measured system.

A quantum system continuously monitored by a quantum limited detector may be assigned, for each individual trajectory, a conditional density operator $\tilde{\rho}_{t}$ that reduces to the usual density operator $\rho_{t}$ when averaged over all of the trajectories, $\rho_{t}=\left\langle\left\langle\tilde{\rho}_{t}\right\rangle\right\rangle[31,35]$. The evolution of $\tilde{\rho}_{t}$ is commonly described by a stochastic master equation that contains a random parameter $\xi(t)$ that accounts for the detector shot noise; see Eqs. (9) and (10) below for an example. An important observation is that such a master equation has a unitary component, corresponding to the dynamics generated by the system's Hamiltonian, and a nonunitary part that stems from the continuous coupling to the detector. For an infinitesimal time step, these two contributions commute and are additive; hence, they may be written as

$$
d \tilde{\rho}_{t}=\delta \mathbb{W}\left[\tilde{\rho}_{t}\right] d t+\delta \mathbb{Q}\left[\tilde{\rho}_{t}\right] d t,
$$

where $\delta \mathbb{W}\left[\tilde{\rho}_{t}\right]$ and $\delta \mathbb{Q}\left[\tilde{\rho}_{t}\right]$ are operators associated with the respective unitary and nonunitary parts of the dynamics. We identify them as corresponding to work and heat at the level of an infinitesimal quantum trajectory. This separation cannot be directly extended to the entire (time integrated) trajectory since the stochastic master equation is generally a nonlinear function of the operator $\tilde{\rho}_{t}$. However, when averaged over quantum fluctuations, Eq. (3) allows us to extend the first law (1) to single realizations of the stochastic measurement outcome,

$$
\begin{aligned}
d \tilde{U}_{t} & =\operatorname{tr}\left\{H_{t}\left(\tilde{\rho}_{t-d t}+d \tilde{\rho}_{t}\right)\right\}-\operatorname{tr}\left\{H_{t-d t} \tilde{\rho}_{t-d t}\right\} \\
& =\operatorname{tr}\left\{\tilde{\rho}_{t-d t} d H_{t}\right\}+\operatorname{tr}\left\{H_{t} \delta \mathbb{W} d t\right\}+\operatorname{tr}\left\{H_{t} \delta \mathbb{Q} d t\right\} \\
& =\delta \tilde{W}_{t}+\delta \tilde{Q}_{t},
\end{aligned}
$$

where, in the second line, $d H_{t}=H_{t}-H_{t-d t}$ and the middle term $\operatorname{tr}\left\{H_{t} \delta \mathbb{W} d t\right\}=0$ since $\delta \mathbb{W}$ originates from the unitary evolution generated by $H_{t}$ itself [36]. In the last line, $\delta \tilde{W}_{t}=$ $\operatorname{tr}\left\{\tilde{\rho}_{t-d t} d H_{t}\right\}$ and $\delta \tilde{Q}_{t}=\operatorname{tr}\left\{H_{t} \delta \mathbb{Q} d t\right\}$, indicating that work is related to a change of the Hamiltonian, as expected, and heat to the nonunitary $\delta \mathbb{Q}$. Equation (4) is a direct extension of stochastic thermodynamics to the quantum domain. The first law (1) is recovered when Eq. (3) is averaged over both stochastic and quantum fluctuations. The integrated work and heat contributions to the changes in transition probabilities, $d \tilde{P}_{m, n}=\tilde{P}_{m, n}^{\tau}-\tilde{P}_{m, n}^{0}$, with $P_{m, n}^{0}=\delta_{n, m}$ being the initial transition probability, can be further obtained from Eq. (3) by carefully adding all of the different terms (see Ref. [37]). We find, for each individual quantum trajectory,

$$
d \tilde{P}_{m, n}=\delta \tilde{P}_{m, n}^{W}+\delta \tilde{P}_{m, n}^{Q},
$$

with the two quantities,

$$
\begin{aligned}
& \delta \tilde{P}_{m, n}^{W}=\operatorname{tr}\left\{\Pi_{m} \int_{0}^{\tau} d t \delta \mathbb{W}\left[\tilde{\rho}_{t}\right]\right\}, \\
& \delta \tilde{P}_{m, n}^{Q}=\operatorname{tr}\left\{\Pi_{m} \int_{0}^{\tau} d t \delta \mathbb{Q}\left[\tilde{\rho}_{t}\right]\right\} .
\end{aligned}
$$

These expressions depend explicitly on the time dependence of $\tilde{\rho}_{t}$ in the whole time interval $[0, \tau]$ (rather than on $\tilde{\rho}_{t}$ and $\tilde{\rho}_{0}$ only), which we stress by using the notation $\delta$ instead of $d$. They provide an unambiguous way to distinguish between work and heat at the level of a single trajectory. Remarkably, they are valid even if the system remains in a coherent superposition of energy eigenstates, that is, when its energy is ill defined. Equation (5) holds for the trajectory averaged quantities $d P_{m, n}=\delta P_{m, n}^{W}+\delta P_{m, n}^{Q}$, with $\delta P_{m, n}^{\alpha}=$ $\left\langle\left\langle\delta \tilde{P}_{m, n}^{\alpha}\right\rangle\right\rangle, \alpha=W, Q$. Because of the nonlinearity of the master equation, this averaged distinction between work and heat contributions to transition probabilities cannot be established solely by the knowledge of $\rho_{t}$, but it requires access to $\tilde{\rho}_{t}$ on single trajectories. It thus cannot be established directly at the ensemble level.

The second law in the form of the Jarzynski equality, $\int d W p(W) \exp (-\beta W)=\exp (-\beta \Delta F)$, immediately follows from Eq. (2) for an isolated system [9]. However, 
the equality is not satisfied for an open system with nonunitary dynamics, owing to the heat term [34]. The second law may be restored by replacing $P_{m, n}^{\tau}$ with $P_{m, n}^{W}=P_{m, n}^{0}+\delta P_{m, n}^{W}$, that is, by setting $\delta P_{m, n}^{Q}$ to zero at each time step; see Fig. 3. We next show how quantum work and heat may be identified theoretically by numerically analyzing a weakly measured two-level system and, experimentally, by means of quantum feedback control.

Application to a monitored qubit.-In order to illustrate our approach, we consider a driven two-level system $S$ with a Hamiltonian, $H_{t}=\epsilon \sigma_{z}+\lambda_{t} \sigma_{x}$, where $\lambda_{t}$ is the external driving and $\sigma_{i}$ the usual Pauli matrices. The system is continuously coupled to a quantum limited detector $D$ via the interaction Hamiltonian $H_{I}$ :

$$
H=H_{t}+H_{D}+\sigma_{z} H_{I},
$$

where, without loss of generality, we identify $\sigma_{z}$ as the system's observable that is monitored by the detector. The effect of the detector is fully characterized by the averaged signals, $\left(I_{1}, I_{2}\right)$, and Gaussian noises, $\left(S_{1}, S_{2}\right)$, measured when the qubit is in the two eigenstates, $(|1\rangle,|2\rangle)$, of the measured observable. We assume this to be in the weak measurement regime, i.e., at time scales smaller than the measurement time $\tau_{M}=\left(S_{1}+S_{2}\right) /\left(I_{1}-I_{2}\right)^{2}$. For concreteness and simplicity, we will interpret the qubit in Hamiltonian (8) as describing a double quantum dot sharing a single electron and interacting with a quantum point contact (QPC), but it can also be applied to a qubit coupled to a microwave resonator [38] in a circuit QED setup as in the experiments [23,24]. We accordingly identify the configurations where the electron occupies only one dot by $\left\langle\sigma_{z}\right\rangle= \pm 1$. Coherent superpositions of the two are possible. The detector monitoring the occupation of the dots is a voltage biased QPC with the Hamiltonian [39-42] $\quad H_{D}=\sum_{l} E_{l} a_{l}^{\dagger} a_{l}+\sum_{r} E_{r} a_{r}^{\dagger} a_{r}+\sum_{l, r} \Omega\left(a_{r}^{\dagger} a_{l}+\right.$ $\left.a_{l}^{\dagger} a_{r}\right)$ and the interaction term reads $H_{I}=$ $\sum_{l, r} \delta \Omega / 2\left(a_{r}^{\dagger} a_{l}+a_{l}^{\dagger} a_{r}\right)$. The signal in the detector is the current $I(t)$ across the QPC, with averages $I_{1(2)}=$ $2 \pi \Omega_{1(2)}^{2} \rho_{l} \rho_{r} e^{2} V / \hbar=e^{2} T_{1(2)} V / h$ and noises $S_{1(2)}=$ $e\left(1-T_{1(2)}\right) I_{1(2)}$. Here, $\rho_{l, r}$ are the densities of states in the left and right electrodes, and $T_{1(2)}$ are the dimensionless transmission probabilities across the QPC.

Under the assumption of a weakly coupled detector, the detector signal is a random variable, and the evolution of the system depends on the specific realization of the stochastic process. This is captured by a well-established Bayesian formalism $[41,42]$ which describes the evolution of the system conditional to the detector's outcome in terms of a nonlinear stochastic differential equation for the system's density matrix $\tilde{\rho}(t)$. In the Ito formulation, we have $[41,42]$

$$
\dot{\tilde{\rho}}_{11}=-2 \frac{\lambda(t)}{\hbar} \operatorname{Im}\left(\tilde{\rho}_{12}\right)+\tilde{\rho}_{11}\left(1-\tilde{\rho}_{11}\right) \frac{2 \Delta I}{S_{0}} \xi(t),
$$

$$
\begin{aligned}
\dot{\tilde{\rho}}_{12}= & 2 i \frac{\epsilon}{\hbar} \tilde{\rho}_{12}-i \frac{\lambda(t)}{\hbar}\left(1-2 \tilde{\rho}_{11}\right)-\tilde{\rho}_{12} \frac{(\Delta I)^{2}}{4 S_{0}} \\
& +\left(1-2 \tilde{\rho}_{11}\right) \tilde{\rho}_{12} \frac{\Delta I}{S_{0}} \xi(t),
\end{aligned}
$$

where $\Delta I=I_{2}-I_{1}$ and $\xi(t)$ is the white noise of the detector's signal, with $\langle\xi(t)\rangle=0,\left\langle\xi(t) \xi\left(t^{\prime}\right)\right\rangle=\sigma^{2} \delta\left(t-t^{\prime}\right)$ and $\sigma=\sqrt{S_{0} / 2}$. The detector current $I(t)$ is, further,

$$
I(t)=I_{0}+\frac{\Delta I}{2}\left(2 \tilde{\rho}_{11}-1\right)+\xi(t) .
$$

For each realization of the measurement outcome, Eqs. (9) and (10) allow us to identify the unitary and nonunitary contributions to the time evolution since the nonunitary part is proportional to $\Delta I$. We rewrite Eq. (3) as $d \tilde{\rho}_{t}=$ $\delta \mathbb{W}\left[\tilde{\rho}_{t}\right] d t+\left(\Delta I / S_{0}\right) \delta \mathbb{M}\left[\tilde{\rho}_{t}\right] d t$, and we identify $\delta \mathbb{W}$ with the work done by the driving $\lambda_{t}$ along an infinitesimal trajectory and $\delta \mathbb{Q}=\left(\Delta I / S_{0}\right) \delta \mathbb{M}$ as the heat associated with the detector backaction of the detector. Because of the nonlinearity of the stochastic master equation, we can only determine the distributions of work and heat numerically. We specify the driving as $\lambda_{t}=g\{1 / \cosh [\nu(1-t / \tau)]\}$, where $\tau$ is the duration of the experiment, and reformulate Eqs. (9) and (10) in the Stratonovich form [41,42]. We solve them numerically by the Monte Carlo method for an ensemble of 300 realizations of the random signal $I(t)$ in the interval $t / \tau \in[0,1]$ using a time step $d t=0.01$. The results for work, heat, and energy along a given quantum trajectory, Eq. (4), are shown in Fig. 1, while those for the work and heat contributions to the transition amplitudes,

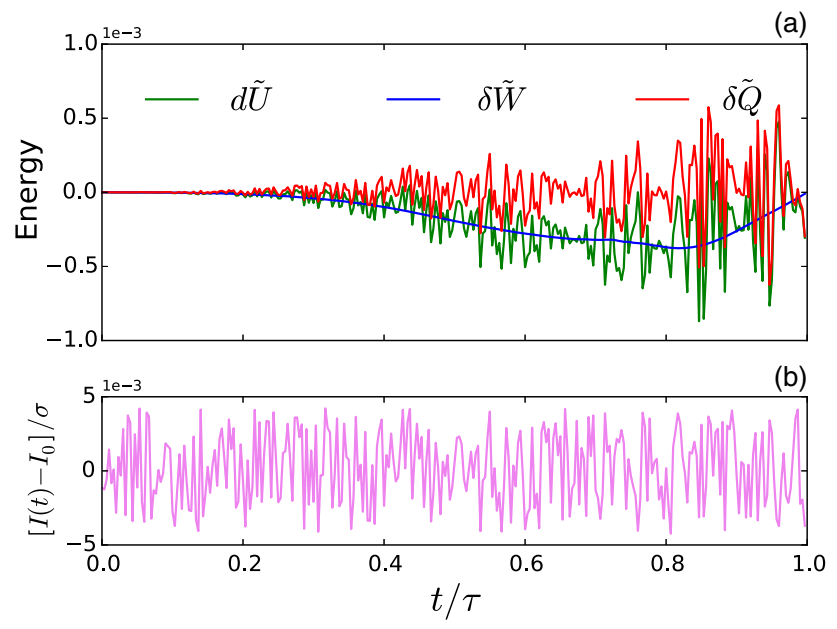

FIG. 1. First law for a weakly measured qubit. (a) Infinitesimal change of work, heat, and energy along a single quantum trajectory $\tilde{\rho}_{t}$; for each realization $d \tilde{U}_{t}=\delta \tilde{W}_{t}+\delta \tilde{Q}_{t}$, Eq. (4). (b) Corresponding signal $I(t)$ in the detector. Parameters are $S_{0} / \Delta I^{2}=2.5 \times 10^{5} \mathrm{dt}, \hbar / g=1.6 \times 10^{2} \mathrm{dt}, \hbar / \epsilon=10^{3} \mathrm{dt}, \nu=8$, and $\tau=3 \times 10^{3} d t$ (see the main text). 


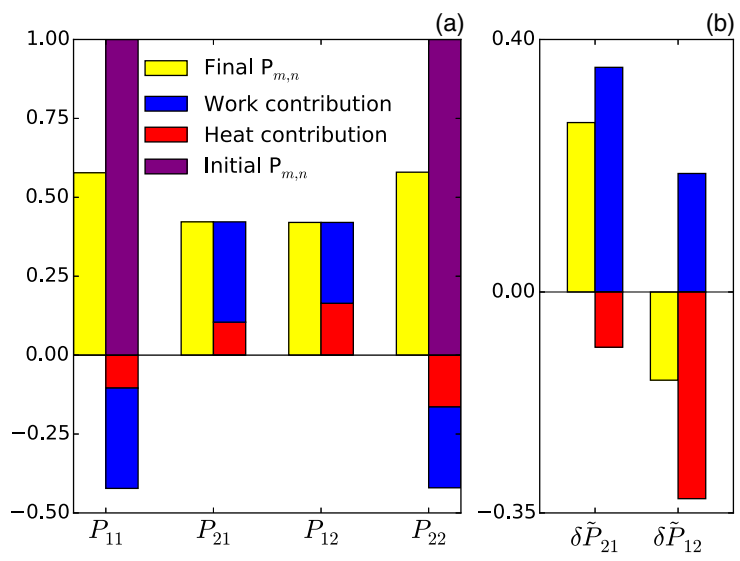

FIG. 2. (a) Averaged final transition probabilities $P_{m, n}^{\tau}$ (yellow) for a continuously monitored qubit with their work and heat contributions, $\delta P_{m, n}^{W}$ (blue), and $\delta P_{m, n}^{Q}$ (red), and the initial transition probability, $P_{m, n}^{0}=\delta_{m n}$ (purple). The first law-like equation $d P_{m, n}=\delta P_{m, n}^{W}+\delta P_{m, n}^{Q}$ is verified. (b) Work and heat contributions $\delta \tilde{P}_{m, n}^{W}$ and $\delta \tilde{P}_{m, n}^{Q}$ at the single trajectory level. Same parameters as in Fig. 1.

Eqs. (6) and (7), are presented in Fig. 2 (see Ref. [37] for details).

Figure 1(a) demonstrates the reconstruction of quantum averaged work and heat changes, $\delta \tilde{W}_{t}$ and $\delta \tilde{Q}_{t}$, along a single quantum trajectory, based on the definitions given in Eq. (4). The corresponding signal $I(t)$ in the detector is displayed in Fig. 1(b). Contrary to the case of an isolated system for which $d \tilde{U}=\delta \tilde{W}$, the heat contribution $\delta \tilde{Q}_{t}$ is clearly visible here. Equation (4) holds for each individual realization and thus extends the first law of stochastic thermodynamics to the quantum regime. Figure 2(b) shows the unambiguous distinction of the work and heat contributions, $\delta \tilde{P}_{m, n}^{W}$ and $\delta \tilde{P}_{m, n}^{Q}$, evaluated via Eqs. (6) and (7), to the final transition probability $P_{m, n}^{\tau}$. We stress that, although $P_{m, n}^{\tau}$ is always positive, as a proper probability should be, the work and heat contributions need not be: the probability to go from state $n$ to $m$ at time $\tau$ can, for instance, be smaller than the initial transition probability [43]. Note that a quantity $d P_{m, n}^{\alpha}=P_{m, n}^{\alpha, \tau}-P_{m, n}^{\alpha, 0}, \alpha=W, Q$ that only depends on initial and final times cannot be defined, reflecting the fact that there are no heat or work operators.

Quantum feedback control.-In classical thermodynamics, work is associated with the variation of the internal energy of the isolated system [1,2]. After having shown above how heat can be theoretically identified, we next take advantage of a feedback loop protocol to suppress the detector backaction [31], offering a scheme to reach isolation experimentally. Quantum feedback has recently been demonstrated experimentally for a superconducting qubit [44]. Specifically, we control the amplitude, $g$, of the system's driving depending on the continuous detector outcome, i.e., $g \rightarrow g_{t} \equiv\left(1-f \Delta \varphi_{t}\right) g$, where $f$ is the feedback strength and $\Delta \varphi_{t}$ the phase difference between the actual vector (with backaction) and the desired vector

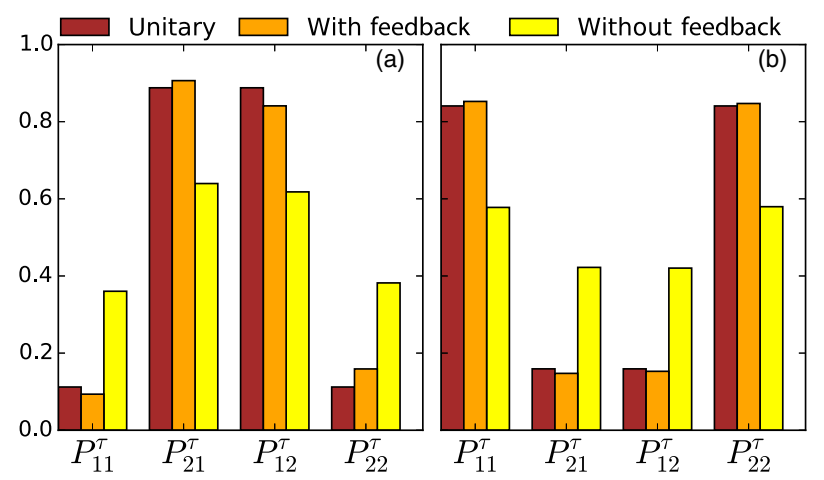

FIG. 3. Transition probabilities $P_{m n}^{\tau}$ for the weakly measured qubit with (orange) and without (yellow) feedback control for (a) $\tau_{1}=1.4 \times 10^{3} d t$ and (b) $\tau_{2}=2.5 \times 10^{3} d t$. The feedback strength is $f=3$. The isolated, unitary case (red) is shown as a reference. The feedback loop effectively suppresses the detector backaction and the associated heat exchange, achieving thermal isolation.

(without backaction) in the Bloch sphere of the qubit (see Refs. [37,41] for details). This allows us to operationally counter the effects induced by the continuous monitoring. From a thermodynamic point of view, the feedback adds an extra amount of work that exactly cancels the heat contribution to the transition probabilities.

Figure 3 shows the numerically simulated final transition probabilities $P_{m, n}^{\tau}$ for the weakly measured qubit with (orange) and without (yellow) quantum feedback for two driving times. We observe in both cases that the feedback process effectively suppresses the heat contributions (identified in Fig. 2) and that the transition probabilities agree with those of the isolated system with unitary dynamics (brown). Quantum feedback control thus appears as a powerful tool to determine the statistics of the work done by the external driving in a continuously monitored system. The heat statistics can be further easily obtained by measuring the undriven system, that is, when no work is performed and $d \tilde{U}_{t}=\delta \tilde{Q}_{t}$.

The above findings can be directly used to verify the quantum Jarzynski equality for the driven qubit. Since any measurement induced heating is prevented by the feedback, only the initial inverse temperature $\beta$ of the system matters. Determining the quantum work statistics via Eq. (2), we find $\Delta F_{1}=-0.488$ and $\Delta F_{2}=-0.496$ with feedback control for $\tau_{1}=1.4 \times 10^{3} d t$ and $\tau_{2}=2.5 \times 10^{3} d t$ and $\Delta F=-0.495$ in the unitary case (for $\beta=10$ ). The excellent agreement demonstrates the correctness of the definitions of work and heat and confirms the second law for a weakly measured quantum system.

Conclusions. - We have extended the laws of stochastic thermodynamics along individual quantum trajectories of a weakly measured system. We have shown how to distinguish work and heat contributions to both the energy changes and the transition probabilities. We have further demonstrated the usefulness of our approach with the 
analysis of a driven qubit and have introduced methods to identify work from heat numerically as well as experimentally with the help of quantum feedback control.

This work was partially supported by DFG under Grants No. RO 4710/1-1 and No. LU 1382/4-1, the EU Collaborative Project TherMiQ (Grant Agreement No. 618074) and the COST Action No. MP1209.

Note added.-Recently, we became aware of a paper by Elouard et al. [45] that also discusses quantum stochastic thermodynamics.

[1] A. B. Pippard, Elements of Classical Thermodynamics (Cambridge University Press, Cambridge, England, 1966).

[2] H. B. Callen, Thermodynamics and an Introduction to Thermostatistics (Wiley, New York, 1985).

[3] U. Seifert, Thermodynamics, fluctuation theorems and molecular machines, Rep. Prog. Phys. 75, 126001 (2012).

[4] C. Jarzynski, Equalities and inequalities: Irreversibility and the second law of thermodynamics at the nanoscale, Annu. Rev. Condens. Matter Phys. 2, 329 (2011).

[5] C. Jarzynski, Nonequilibrium Equality for Free Energy Differences, Phys. Rev. Lett. 78, 2690 (1997).

[6] J. Liphardt, S. Dumont, S. B. Smith, I. Tinoco, and C. Bustamante, Equilibrium information from nonequilibrium measurements in an experimental test of the Jarzynski equality, Science 296, 1832 (2002).

[7] V. Blickle, T. Speck, L. Helden, U. Seifert, and C. Bechinger, Thermodynamics of a Colloidal Particle in a Time-Dependent Nonharmonic Potential, Phys. Rev. Lett. 96, 070603 (2006).

[8] S. Ciliberto, R. Gomez-Solano, and A. Petrosyan, Fluctuations, linear response, and currents in out-of-equilibrium systems, Annu. Rev. Condens. Matter Phys. 4, 235 (2013).

[9] P. Talkner, E. Lutz, and P. Hänggi, Fluctuation theorems: Work is not an observable, Phys. Rev. E 75, 050102 (2007).

[10] G. Huber, F. Schmidt-Kaler, S. Deffner, and E. Lutz, Employing Trapped Cold Ions to Verify the Quantum Jarzynski Equality, Phys. Rev. Lett. 101, 070403 (2008).

[11] M. Esposito, U. Harbola, and S. Mukamel, Nonequilibrium fluctuations, fluctuation theorems, and counting statistics in quantum systems, Rev. Mod. Phys. 81, 1665 (2009).

[12] M. Campisi, P. Hänggi, and P. Talkner, Quantum fluctuation relations: Foundations and applications, Rev. Mod. Phys. 83, 771 (2011).

[13] J. M. Horowitz, Quantum-trajectory approach to the stochastic thermodynamics of a forced harmonic oscillator, Phys. Rev. E 85, 031110 (2012).

[14] F. W. J. Hekking and J. P. Pekola, Quantum Jump Approach for Work and Dissipation in a Two-Level System, Phys. Rev. Lett. 111, 093602 (2013).

[15] R. Dorner, S. R. Clark, L. Heaney, R. Fazio, J. Goold, and V. Vedral, Extracting Quantum Work Statistics and Fluctuation Theorems by Single-Qubit Interferometry, Phys. Rev. Lett. 110, 230601 (2013)
[16] L. Mazzola, G. De Chiara, and M. Paternostro, Measuring the Characteristic Function of the Work Distribution, Phys. Rev. Lett. 110, 230602 (2013).

[17] J. Aberg, Truly work-like work extraction via a single-shot analysis, Nat. Commun. 4, 1925 (2013).

[18] N. Y. Halpern, A. J. P. Garner, O. C. O. Dahlsten, and V. Vedral, Introducing one-shot work into fluctuation relations, New J. Phys. 17, 095003 (2015).

[19] A. J. Roncaglia, F. Cerisola, and J. P. Paz, Work Measurement as a Generalized Quantum Measurement, Phys. Rev. Lett. 113, 250601 (2014).

[20] R. Gallego, J. Eisert, and H. Wilming, Thermodynamic work from operational principles, arXiv:1504.05056.

[21] T. B. Batalhao, A. M. Souza, L. Mazzola, R. Auccaise, R. S. Sarthour, I. S. Oliveira, J. Goold, G. De Chiara, M. Paternostro, and R. M. Serra, Experimental Reconstruction of Work Distribution and Study of Fluctuation Relations in a Closed Quantum System, Phys. Rev. Lett. 113, 140601 (2014).

[22] S. An, J.-N. Zhang, M. Um, D. Lv, Y. Lu, J. Zhang, Z.-Q. Yin, H. T. Quan, and K. Kim, Experimental test of the quantum Jarzynski equality with a trapped-ion system, Nat. Phys. 11, 193 (2014).

[23] K. W. Murch, S. J. Weber, C. Macklin, and I. Siddiqi, Observing single quantum trajectories of a superconducting quantum bit, Nature (London) 502, 211 (2013).

[24] S. J. Weber, A. Chantasri, J. Dressel, A. N. Jordan, K. W. Murch, and I. Siddiqi, Mapping the optimal route between two quantum states, Nature (London) 511, 570 (2014).

[25] A. A. Clerk, M. H. Devoret, S. M. Girvin, F. Marquardt, and R. J. Schoelkopf, Introduction to quantum noise, measurement, and amplification, Rev. Mod. Phys. 82, 1155 (2010).

[26] Y. Aharonov and D. Rohrlich, Quantum Paradoxes (WileyVCH, New York, 2005).

[27] O. Hosten and P. Kwiat, Observation of the spin Hall effect of light via weak measurements, Science 319, 787 (2008).

[28] P. B. Dixon, D. J. Starling, A. N. Jordan, and J. C. Howell, Ultrasensitive Beam Deflection Measurement via Interferometric Weak Value Amplification, Phys. Rev. Lett. 102, 173601 (2009).

[29] A. Romito and Y. Gefen, Weak measurement of cotunneling time, Phys. Rev. B 90, 085417 (2014).

[30] J. S. Lundeen, B. Sutherland, A. Patel, C. Stewart, and C. Bamber, Direct measurement of the quantum wavefunction, Nature (London) 474, 188 (2011).

[31] H. W. Wiseman and G. Milburn, Quantum Measurement and Control (Cambridge University Press, Cambridge, England, 2010).

[32] F. Reif, Fundamentals of Statistical and Thermal Physics (McGraw-Hill, New York, 1965).

[33] A. Peres, Quantum Theory, Concepts and Methods (Kluwer, New York, 1993).

[34] D. Kafri and S. Deffner, Holevo's bound from a general quantum fluctuation theorem, Phys. Rev. A 86, 044302 (2012).

[35] K. Jacobs, Quantum Measurement Theory and its Applications (Cambridge University Press, Cambridge, England, 2014). 
[36] For a unitary process $d \tilde{\rho}_{t}=\delta \mathbb{W} d t=i\left[\tilde{\rho}_{t}, H_{t}\right] d t / \hbar$ and $\operatorname{tr}\left\{H_{t}\left[\tilde{\rho}_{t}, H_{t}\right]\right\}=0$.

[37] See Supplemental Material at http://link.aps.org/ supplemental/10.1103/PhysRevLett.116.080403 for a derivation of the first law along quantum trajectories and for details about the Monte Carlo simulations and the feedback control cycles.

[38] A. N. Korotkov, Quantum Bayesian approach to circuit QED measurement, in Quantum Machines, Ecoles de Physique des Houches Session XCVI, 2011 edited by M. Devoret et al. (Oxford University Press, Oxford, 2014).

[39] S. A. Gurvitz, Measurements with a noninvasive detector and dephasing mechanism, Phys. Rev. B 56, 15215 (1997).

[40] A. N. Korotkov, Continuous quantum measurement of a double dot, Phys. Rev. B 60, 5737 (1999).
[41] A. N. Korotkov, Selective quantum evolution of a qubit state due to continuous measurement, Phys. Rev. B 63, 115403 (2001).

[42] A. N. Korotkov, Noisy quantum measurement of solid-state qubits: Bayesian approach, NATO Sci. Ser., II 97, 205 (2003).

[43] An insightful discussion of the role and interpretation of negative probabilities in physics has been provided by R. P. Feynman, in Quantum Implications: Essays in Honour of David Bohm, edited by F. D. Peat and B. Hiley (Routledge, New York, 1987), p. 235.

[44] K. W. Murch, U. Vool, D. Zhou, S. J. Weber, S. M. Girvin, and I. Siddiqi, Cavity-Assisted Quantum Bath Engineering, Phys. Rev. Lett. 109, 183602 (2012).

[45] C. Elouard, A. Auffèves, and M. Clusel, Stochastic thermodynamics in the quantum regime, arXiv:1507.00312. 